\title{
Building Preservation: The University of Illinois at Urbana-Champaign's Stacks Assessment
}

\section{Thomas H. Teper and Stephanie S. Atkins}

This article discusses the results of two collection assessments conducted at the University of Illinois at Urbana-Champaign. The library's Preservation Committee completed the first assessment and reported its results in College \& Research Libraries in 1989. The second assessment was completed in 2002 and accompanies the institution's initiative to establish the library's first centralized preservation and conservation program. Both assessments focused on the central stacks collection, a repository collection serving the institution's forty-two branch and departmental libraries. Although a reanalysis of the first assessment's data was impossible, the authors attempted to draw comparisons between the two assessments' results. After thirteen years without a preservation program and without any significant facilities improvements, the results provide insight into the results of deferred collections care and facilities maintenance and offer guidance for conducting similar studies with other research library collections.

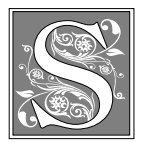

ince the publication of the survey results from Stanford University's Green Library in 1982, the library preservation community has come to rely on collection assessments as a means of learning about the physical state of library and archival collections. ${ }^{1}$ In 1989, the University of Illinois at Urbana-Champaign (UIUC) published the results of its own assessmentLibrary Collection Deterioration: A Study of the University of Illinois at UrbanaChampaign. In the introduction, the authors cited William Barrow's assertion that many of the twentieth century's published books may have a shelf life of less than fifty years. ${ }^{2}$ Written thirty years after Barrow published his research, the authors in Illinois indicated that the reality of this prediction was becoming "painfully obvious" to librarians and other cultural resource managers. ${ }^{3}$ Indeed, the 1989 survey's results indicated that 37 percent of the collection suffered from serious deterioration, 33.6 percent from moderate deterioration, and 29.4 percent from no significant deterioration. ${ }^{4}$

Although an assessment does not provide concrete data about each and every volume, a properly planned and conducted assessment provides a preservation administrator with the means to de-

Thomas H. Teper is the Head of Preservation at the University of Illinois at Urbana-Champaign; e-mail: tteper@uiuc.edu. Stephanie S. Atkins is the Assistant Circulation and Bookstacks Librarian at the University of Illinois at Urbana-Champaign; e-mail: ssatkins@uiuc.edu. The authors wish to thank and acknowledge the Research and Publication Committee of the University of Illinois at Urbana-Champaign Library, which provided support for the completion of this research. 
velop a better understanding of an institution's collections. Indeed, operating under the assumption that preservation funds must be targeted at the areas of greatest need, the value of an assessment in the initial planning of preservation activities and those expenditures is still of paramount importance for maximizing expenditures. Brian J. Baird and colleagues recently reiterated this point in a report on surveys conducted at the University of Kansas:

One of the greatest challenges facing academic and research libraries today is the preservation of collections. The majority of materials housed in research libraries are unique and irreplaceable. Unfortunately, the number of these items needing treatment far exceeds the availability of resources. Therefore, libraries must carefully evaluate their collections and use that information to develop proactive preservation plans. ${ }^{5}$

Spurred by a growth in professional interest in preservation, the University of Illinois began to investigate various means of addressing preservation needs throughout the library in the late 1970s and completed a report in 1980. Another body of individuals completed a statewide analysis project in $1986 .{ }^{6}$ Despite this preparatory work, preservation within both the state of Illinois and the University of Illinois at Urbana-Champaign's library failed to make the headway that it did at other institutions, headway that can be seen in the publication of reports from many institutions completing the Association of Research Libraries' Preservation Planning Program and the continued development of preservation programs at many of these institutions. ${ }^{7}$

Although preservation programs became almost commonplace in many universities and, to a more limited extent, colleges, UIUC's library was unable to develop and maintain a comprehensive preservation program. As a result, the reality that became painfully obvious to many of the institution's librarians in 1989 has been compounded by thirteen years of additional acquisition and deterioration. At the time of the 1989 assessment, the central stacks collection held roughly 5.3 million volumes. Thirteen years and almost 500,000 additional volumes later, the current assessment evaluates possible comparisons between the two data pools and quantifies the collection's physical condition as the library launches a new preservation and conservation program.

\section{UIUC Library and Its Collections}

The University of Illinois at UrbanaChampaign library is nationally and internationally renowned for the depth and breadth of its collections. Its specialized collections are dispersed among forty-two departmental libraries, more than twenty of which are located within the main library building. Currently, the library's total collections number nearly 10 million volumes and 22 million pieces.

With approximately 5.8 million volumes, the central stacks collection is the single largest repository in the campus library system. As collections of currently acquired material grow, departmental libraries transfer older and seldom-used titles into the stacks. As a result, the stacks contain the most diversified and oldest circulating materials in the library.

Unlike many of its peer institutions, UIUC has maintained a closed stack system, granting access only to faculty, graduate students, and a limited number of undergraduates. The result is a circulation model in which many patrons request items, while staff retrieve and reshelve them.

\section{The Central Stacks Collection and Collection Maintenance}

For many years, the library's administration focused primarily on amassing its unique and valuable collection more than on the collection's condition or maintenance. With the notable exception of library binding, rare books and special collections received most available 
preservation and conservation funds. Browsing through the stacks, it is evident that the collection is showing signs of significant deterioration. The stacks office staff stabilized materials, when possible, using boxes and pamphlet binders; and the library's small book repair and pamphlet-binding unit followed suit whenever requested. However, the number of items needing attention exceeded the staff's ability to keep pace with the level of need.

The staff also faces the downside of such comprehensive collection building; the depth and breadth result in large collections of non-Western material and significant collections of semi-ephemeral material. Consequently, the stacks hold the bulk of the library's aging collections and the most significant holdings of materials that continue to be produced on acidic paper-Eastern European, African, and Latin American materials.

The stacks' poor storage conditions exacerbate the problem of aging collections and acidic paper. ${ }^{8}$ Built in 1926, the original building and most of its subsequent additions lack basic air-conditioning and humidity controls. In air-conditioned portions of the stacks, temperatures generally remain relatively stable despite the lack of operational reheat systems. However, those portions not airconditioned often experience extreme fluctuations in temperature and humidity, with ranges from around $60^{\circ}$ to well over $80^{\circ} \mathrm{F}$ and humidity fluctuations between the 30 percent range to over 70 percent in various parts of the building. The combination of high ambient temperature and humidity has resulted in periodic mold outbreaks. Significant, documented mold blooms affected the central stacks collection in 1993 and the Rare Book Room's collection from 1981 to 1987.

The collection also faces the results of decades of campuswide deferred facilities maintenance. Periodic roof and foundation leaks led to limited flooding and subsequent mold outbreaks, including a significant mold bloom in the Rare Book Room during the spring of 2001 and mul- tiple stacks leaks in 2001-2002. In addition, poor air circulation, accumulated dust, and insect infestations have all left their marks on various parts of the collections.

The collection's impressive growth in the second half of the twentieth century has created another significant problemovercrowding. In many areas, the collection's growth has exceeded its shelving capacity to such an extent that books are stacked on the floor in aisles. Presently, the library is in the process of building a high-density shelving facility to alleviate the overcrowding. In preparation for this, the library's administration imposed a moratorium on all departmental library transfers into the central stacks collection, a move that alleviated some pressure on the stacks but added significant pressure to the various branch and departmental libraries.

\section{The Stacks Collection and the Assessment}

The central stacks collection consists of monographs, bound periodicals, and a collection of 82,000 microforms. The microform collection resides in an environmentally controlled room and has recently received some collection maintenance by re-housing a significant portion of the collection. Special formats such as audiovisual materials and digital media are not material types represented in the collection.

The assessment's focus was guided by that of the 1989 assessment. In keeping with the 1989 assessment, specialized collections located within the stacks' physical space were excluded. For example, the stacks' microform collection was not included nor were the government documents collections or the Asian library. Similarly, the assessment excluded unbound periodical titles. This is in keeping with the protocol established by other assessments. ${ }^{9}$ Finally, the assessment excluded a brittle book backlog of roughly 6,000 British and American periodical volumes and monographs dating back to the early nineteenth century. 
Why an Assessment?

Beginning with the publication of Stanford University's assessment of Green Library in 1982 and proceeding until the present, preservation assessments proved themselves a valuable method of informing preservation administrators and collection managers about their collections' condition and enabled them to plan their preservation program's development. ${ }^{10}$ An assessment permits an institution to identify needs and priorities and provides justification for resources.

The authors chose an assessment using random sampling for four primary reasons. First, this enabled some level of comparison between the results of the 1989 assessment and data gathered thirteen years later. Second, because the central stacks collection represents the largest and oldest circulating collection in the library system, assessing this population offered the opportunity to learn about a significant portion of the library's collections.

Third, the imminent construction of a high-density shelving facility on campus influenced the decision to assess the central stacks collection. The facility's first phase will house two million volumes, 100,000 of which are initially being selected from the stacks. This process means that the stacks will no longer exist as currently conceived; in essence, the stacks' current repository function will diminish. Consequently, the authors perceived this to be the last opportunity to conduct such an assessment. Moreover, assessing the central stacks collection will give collection managers and administrators an idea of overall condition before relocating the materials.

Finally, the authors strongly believed that a second, more thorough stacks assessment would be invaluable for long-range preservation planning. Although the 1989 assessment produced useful data about the overall state of the central stacks collection, no data remained pertaining to the individual pieces surveyed. Instead, only the compiled data remained, leaving the in- stitution with no opportunity to reevaluate the original sample. For instance, the 1989 assessment defined only three levels of condition—good, moderate, and poorfor the paper, binding, and boards (table 1). The new assessment enabled the library to document the condition of the collection and to begin the process of thoroughly analyzing needs.

\section{Project Hypothesis}

To ensure the maximum level of objectivity, the assistant circulation and bookstacks librarian and the head of preservation discussed any preconceptions they held about the collection based on their knowledge of the stacks and their understanding of the information gathered by the 1989 assessment. As with the Stanford University assessment published in 1982, the data from UIUC's initial assessment reported in College $\mathcal{E} R e-$ search Libraries employed a system of weighing certain elements of an item's condition in an effort to score overall item condition. ${ }^{11}$ Although this model was effective for gathering the general collection-wide data needed at the time, the sample-level data from 1989 were lost.

Because a direct comparison with the 1989 UIUC study was impossible, the authors designed the new assessment with the purpose of gathering data that were more specific. However, the 1989 assessment did lead the authors to hypothesize that roughly one third of the collection would exhibit signs of significant damage, one third would exhibit signs of mild to moderate damage, and

\begin{tabular}{|c|c|c|c|}
\hline \multicolumn{4}{|c|}{$\begin{array}{c}\text { TABLE 1 } \\
\text { Paper, Binding, And Board and } \\
\text { Cover Condition as Reported in the } \\
\text { 1989 UIUC Assessment } \\
\end{array}$} \\
\hline & Paper & Binding & $\begin{array}{c}\text { Board } \\
\text { and Cover }\end{array}$ \\
\hline Good & $32.0 \%$ & $70.8 \%$ & $49.7 \%$ \\
\hline Moderate & $31.0 \%$ & $24.5 \%$ & $41.9 \%$ \\
\hline Poor & $37.0 \%$ & $4.7 \%$ & $8.3 \%$ \\
\hline
\end{tabular}


the remainder would show no significant signs of damage. Although this is their hypothesis, the authors also understood that multiple factors could have changed this: (1) an increase in the percentage of material published on acid-free paper, (2) patrons' increased usage of electronic resources, (3) the creation of a separate brittle book backlog, and (4) the impact of air-conditioning on a significant portion of the collection.

\section{Statistical Methodology Confidence and Tolerance}

Using proper sampling methodology, a sample of the collection will reasonably estimate the characteristics of any population within a certain margin of error. A confidence level of 95 percent and a tolerance level of \pm 5 percent were acceptable for this study. This requires a sample size of 385 items. This means, for example, that if 32 percent of the books are brittle, the authors are 95 percent certain that the actual percentage of brittle books is between 27 and 37 percent. The earlier survey of the stacks used the same confidence and tolerance levels in its assessment, providing a basis for comparison between the results of the two surveys. Moreover, one downside of a higher level of confidence is the significant increase in the number of items sampled. For example, an increase from 95 to 99 percent requires a nearly 72 percent increase in the number of items sampled-a significant expense relative to the data's potential use for generating broad-based information. Decreasing the tolerance level by one percent increments also would require large increases in the sample size: 56 percent increase for \pm 4 percent; 177 percent increase for \pm 3 percent; and 524 percent increase for \pm 2 percent. This will greatly increase the precision of the results, but, once again, the expense would exceed what additional information the authors can gain by a lower tolerance level.

\section{Sampling Method}

As sample sizes do not increase for populations over 50,000 , a completely accurate estimate of the population was not necessary before beginning the assessment. Consequently, the next step in this project was to determine the sample size required to provide the desired level of accuracy. Rather than using statistical sampling tables, the AZPlanSite provided a calculator that automatically calculated sample sizes based on collection size, maximum acceptable margin of error, and degree of confidence desired. ${ }^{12}$

At the recommendation of a statistician with the campus's Survey Research Lab, the authors chose to ensure that each deck within the stacks had thirty items randomly chosen for the study. This suggestion ensured an adequate representation of the discrete populations within the collection, a situation that stems from the central stacks collection's physical arrangement into seventeen distinct decks. As mentioned earlier, some decks hold collections that are administratively separate from the central stacks collections, resulting in the exclusion of three decks from the sample. Two half-decks were combined into one, as they contained exactly half the number of ranges as the full decks. Thus, the sample assessment consisted of thirteen decks.

Initially, the authors wanted to base the number of samples per deck on the deck's approximate collection size. Unfortunately, determining the exact number of items for each deck was impossible. The stacks have undergone neither a complete inventory nor a full retrospective conversion. Moreover, overcrowding is so severe in some spaces that books are stacked on the floor. Consequently, it is difficult to estimate accurately the number of items located on each deck. With all these limitations known, the statistician recommended that selecting a certain number of items per deck would suffice. Thirty items per deck would be analyzed, achieving a sample size of 390 items. This sample size provides a confidence and tolerance of 95 percent \pm 5 percent.

To locate items within the collection, the authors used Microsoft Excel's random number generator to provide a single num- 
ber that corresponded to each range within the stacks. Three numbers were generated to locate each column and each shelf. Six numbers were generated for each book. Variations in the number of columns, shelves, and books per shelf necessitated the generation of multiple numbers. These provided a backup for locating items when there was no corresponding column, shelf, or book. Student employees received fifty samples each and instructions to stop when they had analyzed thirty books on each deck. These additional samples ensured that rejects due to insufficient columns, shelves, and books per shelf would not interfere with sampling the necessary number of items per deck.

\section{Biases}

Although the authors made every effort to include as much of the collection as possible within the sample, the necessary exclusion of certain collection materials means that some biases exist in the study.

- A 6,000-volume brittle book backlog was not considered within the assessment.

- No satisfactory manner was devised to include the books currently stacked on the floor. The authors gave significant consideration to these "nonrespondents." Consultation with a statistician produced no better solution than including these materials within a broad category of biases. ${ }^{13}$

- The number of shelving units varied depending on the deck, ranging from 229 to 387 . Because the same number of samples was set for each deck, the materials on the decks with the lowest number of ranges had a greater probability of selection than did materials on decks with the highest number of ranges. However, this method guaranteed sampling from all portions of the collection.

- Most of the collections reside in portions of the stacks without air-conditioning. However, the newest, high-density addition to the stacks does receive central air. Nearly 45 percent of the items sampled came from the decks in this addition. Although shifting and additions have been made in this area, these conditions could make a difference in the collections housed here.

- The number of columns and shelves varies depending on book size. The folio cabinets have fewer columns and shelves than standard regular ranges. Thus, they likely constitute a higher number of rejections. The original assessment used a similar method to generate random numbers, and the authors chose to duplicate the process, recognizing that the results may not reflect exactly how many items in the collection are folios.

\section{Work Flow, Labor Management, and Instruction}

The assistant circulation and bookstacks librarian hired five student employees to conduct the study. At the time, it was not feasible to reallocate central circulation and preservation staff to work on the study. Instead, funding opportunities were available from sources within the library to use student employees for research projects, and the authors received a grant to hire students for the study.

In preparation for the project, the students were required to attend a single training program consisting of an overview of the project's goals, an introduction to book construction, and a discussion of assessment techniques and methods. Through a Microsoft PowerPoint presentation, images illustrated a variety of different types of cover and paper damage, as well as some basic binding information. Each student received a copy of the PowerPoint presentation for reference. This portion of the workshop concluded with the students analyzing books and presenting their findings to the other students. This hands-on exercise provided an opportunity to test what they learned and enabled the authors to emphasize and clarify certain details.

The workshop included a tour of the stacks to familiarize the students with their arrangement and the method for locating materials using maps and the random numbers. At the end of the tour, the assistant circulation and bookstacks librarian 
gave each student a set of random numbers and a map and instructed the students to find the correct book on the deck. Only one student out of five had trouble finding the correct book on the shelf, and after a thorough explanation, the librarian was satisfied that the students had a good grasp of how to use the random number sets and the maps. On the random sample form, the students noted the reason why a random number set was rejected (e.g., insufficient number of books on the shelf). The librarian reviewed the reasons for each rejection to ensure that no sets were discarded in error.

Instruction did not end with the workshop and tour. The students regularly communicated with the authors and asked a variety of questions, ranging from finding the date of publication to identifying types of damage. The authors also reviewed completed forms and discovered that the students had a good eye for detail. However, some students were initially too quick to note damage when none was present. The authors spoke to the students to clarify differences (i.e., yellowed paper versus the natural color of the paper) and the students corrected their forms. In total, the students took eighty-three hours to analyze 390 books, averaging thirteen minutes per book. For books that the students required consultation to complete, the assessment process took longer than thirteen minutes, but for books with minimal or no damage, students needed only a few minutes to fill out the form. One of the reasons why the students were efficient in assessing the books was that all of their samples were on two to four decks. The students became intimately familiar with the layout and peculiarities of their assigned decks and were able to navigate with ease.

When all forms were completed, a student entered the data from the assessment into a relational database. The preservation librarian examined the forms for discrepancies or typos before the data were inputted into the database. After being entered, the data again were reviewed for possible errors or omissions.

\section{The Assessment Results}

In the most basic terms, the results from the first assessment corroborated those provided by the second assessment. In terms of brittleness, 35.64 percent of the pieces sampled in 2002 exhibited some degree of embrittlement, as opposed to 37 percent in 1989. Similarly, 29.2 percent of those pieces sampled in 1989 suffered from moderate or poor binding conditions. ${ }^{14}$ In 2002, those with detached boards, loose hinges, tears, or missing covers accounted for 24.88 percent of the collection. Given the tolerance of $\pm 5 \%$, the data initially suggested no significant differences between the populations.

As noted earlier, the current assessment was divided into four basic sections: a brief examination of publication information, an examination of the binding, an assessment of any enclosures, and an assessment of the text block that focused on damage to the pages.

\section{Publication Information}

To develop a better understanding of the collection, the assessment gathered data about the publications' bibliographic history. This included data on the relative size, date, and place of publication.

\section{Size}

Of the 390 pieces examined, 79.23 percent of the collection was of a standard size, measuring less than ten and a half inches and over six inches in height. This is equal to nearly 4.6 million volumes from the entire collection of nearly 5.8 million pieces. Undersized material equaled 2.82 percent of the collection, and oversized material equaled 15.9 percent. Another 2.05 percent of the pieces reflected folios requiring flat shelving. ${ }^{15}$

\section{Date of Publication}

As one would expect with such a representative sampling method, a year-byyear analysis of publication dates yields little substantive data about the entire collection. However, analyzing the data by decade of publication does present an interesting illustration of both the 
collection's development and composition. Although a separate project began isolating those items in the central stacks collection published before 1800 , the assessment indicates that nearly 74,202 volumes were published before the beginning of the nineteenth century. As part of the selection for high-density shelving, rare book and special collections librarian is reviewing early imprints for transfer to the Rare Book Room.

The collection's growth from 1870 onward appears to be almost unchecked (table 2). Until 1980, its rate of growth increased nearly every decade with the exceptions of the decades 1881-1890 and 1941-1950. The first of these decades follows a period of economic depression in the early 1870s and actually precedes the depression that accompanied discussions about abandoning the gold standard in 1890. The second decade encompasses the Second World War. Whether either of these periods of diminished growth can be directly attributed to diminishing resources or decreasing book production is unlikely. However, it is likely that both played a role.

From 1981 to the present, the rate of growth appears to have declined. However, the system comprises forty-two subject and departmental libraries. With the exception of Slavic and Eastern European, Latin American, and European blanket orders, many core titles and the most current acquisitions are not sent to the central stacks collection. Consequently, the vast bulk of volumes acquired since 1981 are still housed in departmental libraries, meaning that the significant drop in collection breadth during this period does not necessarily indicate a decline in the breadth of the overall collection.

The collection of material published in the decades before the university's incorporation in 1867 is more difficult to generalize. Lower numbers of these items within the stacks resulted in lower populations for each of these decades. For example, imprint dates from the first three-quarters of the nineteenth century indicate that the collection ranges from lows of 14,864 volumes to highs of 74,321 volumes per decade.

\begin{tabular}{|cc|}
\hline \multicolumn{2}{|c|}{ TABLE 2} \\
Date of Publication as Reported \\
in the 2002 Assessment
\end{tabular}

\section{Place of Publication}

Predictably, North American publications constitute the largest single portion of the collection, as shown in table 3. At 42.82 percent, this represents nearly 2.5 million volumes. This is closely followed by the combined weight of roughly two million Western European volumes and nearly threequarters of a million Eastern European volumes. The most surprising result is that 4.1 percent of the sampled volumes trace their origin to India or other parts of Asia. The Asian library is one of the few libraries that does not transfer collections into the stacks; however, many other libraries purchase materials published in these areas and include them within their collections. Moreover, many of these items are received through the PL480 program and the Farmington Plan. 


\section{Binding Information}

\section{Binding Style}

Examination of the binding styles focused on determining which of eight choices best characterized the type of binding represented by each piece. In general, the definitions were clear to most of the student employees. The one area that required some additional definition was the relationship between library binding and leather binding. For the purposes of this assessment, a library binding consisted of any binding performed by a binder after the library had purchased the piece. These items ranged from early quarter- and halfbound pieces with marbled boards to contemporary library bindings. A leatherbound volume was a volume bound entirely in leather presumed to have been sold in that binding and generally monographic in nature. With 43.33 percent of its collection library bound, 1.28 percent of its material in full leather, and less than a third of the collection in an original publisher's cloth binding, these results suggest that the library has relied heavily on library binding as both a management and maintenance tool (table 4).

\section{External Cover Damage: Hinges and Mechanical Construction}

An examination of the mechanical construction of the volumes' covers indicated that 24.88 percent \pm 5 percent of the col-

\section{TABLE 3}

Place of Publication as Reported in the 2002 Assessment

\begin{tabular}{lrr} 
Category & \% of Total & \multicolumn{1}{c}{$\begin{array}{c}\text { Total } \\
\text { Pieces }\end{array}$} \\
\hline N. Amer. & 42.82 & $2,482,305$ \\
L. Amer. & 6.67 & 386,665 \\
W. Europe & 33.59 & $1,947,236$ \\
E. Europe & 12.56 & 728,112 \\
India & 1.79 & 103,768 \\
Africa & 0.26 & 15,072 \\
Aus/NZ & 0.00 & 0 \\
Asia & 2.31 & 133,912 \\
\hline Total & 100.00 & $5,797,070$ \\
\hline
\end{tabular}

TABLE 4

Binding Style as Reported in 2002 Assessment

\begin{tabular}{lcc}
\hline \hline Category & Number & \% of Total \\
\hline Publishers' cloth & 123 & 31.54 \\
Library binding & 169 & 43.33 \\
Softcover & 47 & 12.05 \\
Velobind/comb & 3 & 0.77 \\
Spiral & 0 & 0.00 \\
Pamphlet binder & 34 & 8.72 \\
Other binding & 8 & 2.05 \\
Vellum binding & 0 & 0.00 \\
Leather & 5 & 1.28 \\
Unbound & 1 & 0.26 \\
\hline Total & 390 & 100.00 \\
\hline
\end{tabular}

lection exhibited some signs of damage, including one or more of the following conditions: detached boards $(3.33 \%)$, loose hinges $(13.33 \%)$, tears $(9.23 \%)$, and no boards or covers whatsoever $(2.56 \%)$. In terms of pure numbers, this means that approximately 341,447 items suffer from either missing or detached boards.

When compared to the 29.2 percent of items indicated as exhibiting poor or moderate binding condition in the 1989 assessment, it is interesting to note that the items currently suffering from detached and missing boards vary from the original data by only 4.32 percent, within the margin of error outlined by the earlier assessment. Similarly, the combined percentages of those covers with tears and loose hinges equal 22.56 percent of the collection. In the 1989 assessment, 24.5 percent of the bindings exhibited "moderate damage."16

\section{External Cover Damage: Other Damage}

Another component of the assessment was damage to the cover of a piece. Any item with visible cover damage, such as water damage, warped and misshapen boards, light bleaching, staining, and insect damage, was counted as one with external cover damage. The total percentage of sampled items without any visible external damage of this type is 43.59 per- 
cent. Examination of the other types of visible damage indicates that there are some significant problems with materials and their storage environment. Two areas of particular concern are materials suffering from combinations of visible water damage, staining, and misshapen covers and materials exhibiting significant abrasion.

In general, the quantity of items with both water damage and staining is high. Approximately 3.59 percent of the materials sampled are water damaged or stained. Although this percentage does not sound excessive, it does indicate that roughly 208,115 pieces have been exposed to leaks, spills, accidents, or other mishaps in their history. This damage may have happened while the pieces were in departmental libraries or in circulation or before they became a part of the library's collections; however, it does indicate that facilities problems and care and handling issues are taking their toll.

Although multiple mold outbreaks have occurred within the stacks, some affecting several deck levels simultaneously, none of the sampled materials exhibited visible evidence of external mold damage. There are a couple of possible explanations for this potential discrepancy. Cleaning projects followed several of the mold outbreaks. Moreover, some pieces may have been damaged to such an extent that they were rebound, resulting in no visible signs of damage to the cover. Another explanation is that the students did not assess the damage correctly and mistook dormant mold for dirt or other debris. Because no systematic cleaning of the stacks has taken place, items with mold may not be easily identifiable from other dusty volumes. Finally, some items marked as stained may, in fact, be showing signs of mold damage.

The other area of concern is the quantity of misshapen pieces. Although evident when walking through the stacks, the results of improper shelving, the improper use of bookends, and overly tight shelving are more striking when the damage is quantifiable. The 2002 assessment indicates that roughly 16.15 percent of the collection is misshapen. This translates into 936,227 pieces permanently marred through mishandling, poor stewardship, and overcrowding. When simultaneously calculated with those items that are both scratched and misshapen, 4.1 percent of the collection, or approximately 237,680 pieces, exhibits this type of compound damage.

\section{Extraneous Material}

The amount of extraneous material associated with the covers of sampled items is relatively low. In general, it is reassuring to see that few pieces received improper repairs using adhesive or cloth tape. In this case, the lack of regular care has proved itself a benefit in terms of long-term care. The total number of items with tape or other extraneous material applied to the cover total only 4.36 percent.

\section{Enclosure Information}

In general, the assessment produced very little information about the enclosures present throughout the collection. Indeed, only one piece of the 390 sampled had any type of enclosure. It was a phase box, and it, incidentally, suffered from some minor damage itself. This figure represents 0.26 percent of the collection, or 15,072 volumes.

At first glance, this low number may indicate that few pieces actually need this type of enclosure; however, the small number of enclosures also can reflect two very different conclusions. As mentioned earlier, the "storage collection" was not included in the sampled pieces. It represents a backlog of severely embrittled pieces. This collection ceased growing in the mid-1990s. Consequently, it is likely that a significant portion of the collection that should have been boxed was put into this holding area.

The second conclusion that one may draw from the low number of enclosures is the relatively low level of past preservation efforts. With about 3.33 percent of the collection suffering from detached 
boards and over 17 percent of the collection brittle to the point that the paper cannot withstand a single double-fold (table 5), there is likely a significant backlog of material that will, at minimum, likely benefit from an enclosure.

\section{Internal Pages Leaf Damage}

Actual paper damage is best represented by noting that 159 of the 390 sampled items exhibited no signs of damage. This represents 40.77 percent of the collection, or about $2,363,465$ volumes with no internal damage. Of those exhibiting one or more categories of leaf damage, the reasons are likely varied; however, staining and water damage often result from the same event.

Perhaps the most striking concern raised is the number of volumes indicating evidence of marking (as characterized by highlighting and underlining) and/or staining. Nearly 11 percent of the sampled items were obviously marked. The library has a long-standing history of encouraging access both on campus and off, a tradition that is valued and unlikely to change in the near future. However, the high incidence of marking is indicative of users' poor care and handling practices. Ten percent of the sampled pieces suffered from some sort of staining. The staining was the result of a variety of sources, but only a small portion of the stained items also visibly suffered from other related forms of damage, including water, visible mold, and insect damage. One conclusion is that many of these other stains resulted from user-related damage.

Of additional concern is the 3.08 percent of volumes (or 178,550) that exhibit some sign of pest-related damage to the text block. Although this may include damage that transpired before their accession by UIUC, this evidence indicates that some contemporary damage is occurring.

\section{Extraneous Material}

As would be expected, a number of volumes suffered from the presence of various extraneous materials. Slightly less

\begin{tabular}{|lc|}
\hline \multicolumn{2}{|c|}{ TABLE 5 } \\
\multicolumn{2}{|c|}{$\begin{array}{c}\text { Acidity to Breakability as } \\
\text { Reported in 2002 Assessment }\end{array}$} \\
\hline \hline Category & \% of Total \\
\hline Acidic/One Fold & 17.18 \\
Acidic/Two Folds & 8.72 \\
Acidic/Three Folds & 4.87 \\
Acidic/Four Folds & 4.87 \\
Acidic/Not Brittle & 54.62 \\
Non-acidic/One Fold & 0.26 \\
Non-acidic/Two Folds & 0.26 \\
Non-acidic/Three Folds & 0.00 \\
Non-acidic/Four Folds & 0.00 \\
Non-acidic/Non-Brittle & 9.23 \\
\hline
\end{tabular}

than 4 percent of the collection had adhesive tape affixed to leaves, likely in an attempt to repair damaged pages. In terms of patron-deposited materials, 6.41 percent had paper slips inserted into text blocks, 1.28 percent had paperclips on leaves, and another 1.03 percent had selfadhesive notes applied to pages. Approximately 3.33 percent of the collection had other extraneous material inserted within the text blocks. Unfortunately, the assessment did not include provision to gather data on the types of extraneous material found.

\section{Paper Acidity}

As the repository for much of the library's older materials, it stands to reason that the stacks collection would suffer from a higher-than-expected level of paper acidity. Indeed, that is the case. Of the 390 items sampled using an Abbey $\mathrm{pH}$ pen, 352 tested acidic on the exterior margin of the last page of text. Even though this measures edge acidity and not core acidity, the result indicates that 90.26 percent of the central stacks collection is acidic. In terms of actual numbers, this translates into approximately $5,232,435$ pieces from a population totaling $5,797,070$.

\section{Paper Acidity as Related to Brittleness}

As was demonstrated in the earlier subsection on date of publication, approximately 
TABLE 6

Paper Acidity to Brittleness as Reported in 2002 Assessment

\begin{tabular}{lccc}
\hline Category & Number & \% of Acidic Books & \% of Total Collection \\
\hline Acidic \& Not Brittle & 213 & 60.51 & 54.62 \\
Acidic \& Brittle & 139 & 39.49 & 35.64 \\
\hline Total & 352 & 100.00 & 90.26 \\
\hline
\end{tabular}

80.26 percent of the central stacks collection was published before 1980 . Nearly all of these were published on acidic paper. As noted earlier, only a portion of the central stacks is air-conditioned. Indeed, nearly 55 percent of this collection resides in space completely governed by the ambient air temperature. Consequently, the collection would be expected to suffer from a high level of embrittlement.

Of those portions of the collection printed on acidic paper, a significant percentage suffers from some level of embrittlement. As shown in table 6, 60.51 percent of the acidic books are not yet brittle whereas 39.49 percent are suffering from some level of embrittlement. In terms of the entire collection, these figures translate into 54.62 and 35.64 percent, respectively, of the entire collection. That means that the stacks collection is composed of nearly 2,066,076 volumes that are acidic and brittle and another 3,166,360 volumes that are acidic, but not yet embrittled. Although some older volumes constructed of higher-quality papers will remain pliable despite high levels of acid, the vast majority of the volumes that are acidic, but not yet brittle, will likely continue to degrade and become increasingly fragile.

\section{Paper Acidity as Related to Breakability}

Even though the assessment determined that 35.64 percent of the entire collection is both acidic and brittle, planning for long-term collection maintenance activities requires a more detailed analysis of the pieces themselves and their ability to withstand other forms of treatment.

In terms of treating this collection, the relation of acidity to embrittlement level is crucial in planning for long-term collec- tion maintenance activities. Of the 139 pieces that were both acidic and brittle, the paper's functionality varies greatly. A total of 17.18 percent of the sampled pieces were both acidic and embrittled to such a point that the paper could not withstand one double-fold without breaking (table 5). Acidic pieces that could withstand two folds accounted for 8.72 percent of the entire collection, and acidic pieces that could withstand three to four folds accounted for 9.74 percent of the collection.

Of additional interest is the fact that the library completed an air-conditioned stacks addition in $1984 .{ }^{17}$ Those items in the new addition were located in climatecontrolled environment for only five years before the 1989 survey was completed. At the time of the original survey, the change in the collection's environment had only a marginal impact on the state of the collection. However, because eighteen years have passed since the new addition was completed, the authors conducted a preliminary investigation to determine the effects of superior environmental conditions on the state of the collection in the new addition.

Of those items sampled in the addition, the number published on acidic paper nearly equaled the results for the entire sample population. However, the number of items that were both acidic and brittle is significantly reduced. For example, only 11 percent of this subpopulation broke on one double fold, compared to 17 percent from the entire sample population. Similarly, only 4.3 percent of those items located in better environmental conditions broke on two double folds, compared to 8.72 percent from the entire population. This trend continues throughout the entire brittle component 
of this population. Some of the difference is certainly due to the acquisition of nonacidic materials, but the possibility exists that this may be, in part, from eighteen years in better storage conditions. However, more comprehensive research is needed to determine this conclusively.

\section{Acidity to Decade of Production}

The assessment tool used to manage the sample also permitted the authors to generate data relating the decade of production to a publication's acidity. From analyzing these data, it is possible to see the distribution of acidic to nonacidic books by decade of publication. As data from the current assessment demonstrate, the percentage of acidic volumes begins to drop off in 1981-1990, the decade that includes the widespread introduction of acid-free paper (table 7). However, it is not until the next decade that the number of nonacidic publications begins to have a significant impact on the relative number of acidic publications in the collection.

With the current assessment in hand, the library now has a unique opportunity to begin to address its history of benign neglect.

\section{Assessment Conclusions and Programmatic Development}

Recognizing that the collection has suffered from the lack of regular maintenance, the University of Illinois at Urbana-Champaign seriously began to address preservation concerns in the past few years. Two years ago, UIUC hired a consultant to complete a needs assessment. Primarily focusing on the facilities, the consultant's report gave the institution the ability to tangibly discuss need. For example, in addition to highlighting the deficiencies caused by deferred maintenance, the assessment provided the library with an estimate of the collection's replacement value. ${ }^{18}$ Although this type of information does not immediately address concerns, it has been valuable in lobbying the administration and increasing awareness of the library's value to the campus.
In addition, the library hired a preservation librarian and a conservator to begin the planning and implementation of a preservation program. Coupled with a significant grant from the Andrew F. Mellon Foundation to outfit and staff a conservation lab in its high-density shelving facility, the program is making great strides in meeting the needs of such a large and varied collection.

However, this does not address how this assessment will shape the preservation and conservation program's overall development. Broadly viewed, the data generated can be grouped into three areas of general need and/or utility: education and outreach; collection repair and maintenance; and administrative development.

\section{Education and Outreach}

As evidenced by Jeanne M. Drewes and Julie A. Page's Promoting Preservation

\begin{tabular}{|lc|}
\hline \multicolumn{2}{|c|}{ TABLE 7} \\
Acidity to Date of Production as \\
Reported in 2002 Assessment \\
\hline \hline \\
Decade & \% Acidic \\
\hline Pre- 1800 & 80.00 \\
$1801-1810$ & 100.00 \\
$1811-1820$ & 50.00 \\
$1821-1830$ & 100.00 \\
$1831-1840$ & 100.00 \\
$1841-1850$ & 100.00 \\
$1851-1860$ & 100.00 \\
$1861-1870$ & 100.00 \\
$1871-1880$ & 100.00 \\
$1881-1890$ & 100.00 \\
$1891-1900$ & 87.50 \\
$1901-1910$ & 100.00 \\
$1911-1920$ & 100.00 \\
$1921-1930$ & 100.00 \\
$1931-1940$ & 100.00 \\
$1941-1950$ & 93.75 \\
$1951-1960$ & 95.12 \\
$1961-1970$ & 98.15 \\
$1971-1980$ & 92.06 \\
$1981-1990$ & 77.78 \\
$1991-2000$ & 53.13 \\
$2001-P r e s e n t$ & 50.00 \\
Unknown & 85.71 \\
\hline
\end{tabular}


Awareness in Libraries: A Sourcebook for Academic, Public, School, and Special Collections, education and outreach have a long tradition within the preservation program arsenal. ${ }^{19}$ This education generally takes multiple forms, targeting both employees and users. Based on the significant number of volumes suffering from highlighting, underlining, and other markings $(637,678)$ or from the use of inappropriate bookmarks such as paperclips and self-adhesive notes $(74,202$ and 59,710, respectively), neither the library's users nor its employees have been educated about the damaging effects of such items or the importance of removing them when the volumes are returned.

Moreover, the fact that significant portions of the collection's external cases are scratched $(1,233,616)$ and misshapen $(936,227)$ indicates that significant care and handling issues have not been addressed. This may include poor handling by users, but it also likely includes the deleterious effects of overly tight shelving. Even though it is expected that the library's planned high-density shelving facility will resolve much of this issue, the fact remains that there are serious deficiencies in basic care and handling education.

Finally, a significant portion of the collection suffers from water damage and staining. Although it is likely that leaks and other faults in the building structure have caused much of this damage, some of it is the result of careless handling or untreated damage.

To address some of these issues, the preservation and conservation program has taken a three-pronged approach. Internally, the head of preservation, the conservation librarian, the associate university librarian for collections, and a member of the repair unit have begun to develop and hold a series of educational workshops for employees. Thus far, the program consists of a preservation orientation, training in library binding, and training in basic book repair. Additional workshops on disaster response and preservation decision making for collection managers are planned for the future.
In addition, the head of preservation initiated a silent campaign of raising preservation awareness among both employees and users. Several months ago, the program began producing a periodic newsletter that provides basic information on the preservation program and its offerings, how to take better care of collections, and preservation in general. Distributed electronically, the newsletter also includes a What's This? section with a picture highlighting either examples of preservation do's and don'ts or items of interest such as images of high-density storage facilities in operation.

Efforts to reach the library's user population are still developing. However, through a collaborative project with a member of the university's faculty and a senior graphics design class, the preservation and conservation program now has designs for posters, bookmarks, and screensavers to communicate the preservation message.

\section{Collection Maintenance and Repair}

Although the library's bookstacks office and book repair unit have traditionally completed repairs, establishing the preservation and conservation program has focused heavily on developing these capacities to better serve the institution's needs. The program's first year and a half focused primarily on the improvement and rationalization of existing services. For example, before September 2001, there was no formal relationship between library binding and book repair. Units with damaged materials sent them to either book repair or binding, generally without instruction and frequently without knowledge of actual need. Consequently, the library outsourced to a library binder many repairs that could have been done by existing internal staff and completed repairs on many items that should have been rebound.

Similarly, many items did not receive the more in-depth treatment their damage warranted. As evidenced by the library's long-neglected backlog of brittle materials, the treatment of brittle materi- 
als was scattered and infrequent. Some items received phase boxes and others various inappropriate or outdated repairs, many of which caused permanent damage to the original items. To address this issue in a more comprehensive manner, the library's administration authorized the new position of a brittle books coordinator. Working with the head of preservation, the brittle books coordinator is establishing the institution's first comprehensive replacement and reformatting program, both digging through the backlog and dealing with those materials that are now being routed to the preservation and conservation unit.

In addition to tackling damaged materials, the preservation and conservation program began to systematically address the issue of binding paperback acquisitions. The assessment indicates that, of the entire sample population, forty-eight items, or 12 percent of the sampled items, appeared in soft covers. Excluding those that the library had bound at some point, nearly 39 percent of the sampled items produced since 1989 are paperbacks. In the long run, this indicates that the library's collection patterns are changing significantly and that its binding patterns need to follow suit. Assessment results indicate that 4.2 percent of all paperbacks sampled suffer from damaged or mended leaves and that an additional 4.2 percent have covers mended with either cloth or adhesive tape. Even though this equals only one percent of the entire sample, it implies that roughly 58,000 paperbacks have received or need repairs. In addition, eleven of the paperbacks sampled were misshapen. This only equals 2.8 percent of the entire collection but amounts to a startling 23.4 percent of all paperbacks sampled. Using these data, the preservation and conservation program has begun to implement a systematic means of addressing the binding needs of its paperback collections.

\section{Administrative Development}

The assessment's most far-reaching outcome is the ability to provide the institu- tion with concrete data for administrative development. As mentioned earlier in this article, the campus has followed a longterm policy of deferred facilities maintenance. The site survey performed by a consultant in 2001 provided the campus and the library's administration with a common language when discussing the collection-its monetary value to the campus. This assessment follows suit by providing the library's administration with the ability to justify improvements based on specific needs.

For example, the 1989 assessment concluded that 37 percent of the collection's paper quality was poor. ${ }^{20}$ At the conclusion of the assessment described in this article, the authors can assert that slightly over 35 percent of the collection is composed of paper suffering some level of embrittlement. Although this is generally in keeping with the 1989 assessment, there is both good news and bad. The good news is that although 35 percent of the collection is embrittled, only 25.9 percent is on acidic paper that fractures after one or two double-folds. Another 0.52 percent of the sampled items are printed on nonacidic paper that is likely coated and breaks after two or fewer folds. The bad news is that the combination of these numbers indicates that the institution is in possession of roughly $1,531,586$ extremely brittle volumes within this single collection.

Despite this significant corpus of embrittled materials, the more shocking figure is that a further 54.62 percent of the collection is printed on acidic paper that has not yet become embrittled. Consequently, the library is in possession of a collection composed of 90.26 percent acidic paper in a facility with extremely fluid environmental conditions with another 0.52 percent that, although acid free on the surface, is very fragile. These data illustrate that, untreated, the rate of deterioration is having an impact that presently outpaces the rate of acid-free transfers into the collection. Despite the significant increase in the acquisition of acidfree pieces in the past two decades, the 
percentage of embrittled pieces is remaining relatively stable. This could stem from a number of reasons, including the continued rate of deterioration in an unstable environment, an increased rate of acquisition of older materials through the institution's robust gifts program, or the delay in the transfer of more current acidfree acquisitions into the central stacks. Regardless of the cause for such continued deterioration, the data in table 7 indicate that the production of acid-free paper will have a significant impact on the long-term health of our collection. However, the continued deterioration of our older collection items indicates that the long-term effects of acid-free paper are not likely to be seen for some time when considering larger collection-wide preservation issues at UIUC.

The ability to contrast the replacement and reformatting or deacidification needs of this collection against the significant costs of introducing environmental controls is valuable for the library's administration. Despite the addition of nearly 250,000 acid-free publications to this collection since 1989, the institution is just keeping pace with the overall rate of embrittlement. Although the cost of proper HVAC controls is staggering, it is dwarfed by the costs of replacement, reformatting, and deacidification. If the possibility exists that improved conditions did slow the rate of embrittlement, this assessment's results provide the library's administration with a significant tool in its quest to improve the facility's conditions.

Within the library, the data provided by this assessment are guiding programmatic development in some significant ways. As noted earlier, the preservation and conservation program has been making strides in improving and expanding services. This development also has included the development of policies and procedures that seek to address internal deficiencies. Examples include the development and phased implementation of uniform binding criteria and efforts to increase awareness among individual employees about their stewardship role. Although these efforts are meeting varying levels of success in such a large system, they are steps in the library's long process of developing a modern and responsive preservation and conservation program.

\section{Notes}

1. Sara Buchanan and Sandra Coleman, "Deterioration Survey of the Stanford University Libraries Green Library Stack Collection," in Preservation Planning Program Resource Notebook, comp. Pamela W. Darling (Washington, D.C.: Association of Research Libraries, 1987), 159-230.

2. Tina Chrzastowski, et al., "Library Collection Deterioration: A Study at the University of Illinois at Urbana-Champaign," College E Research Libraries 50 (Sept. 1989): 577-84. Barrow's assertion is available at W. J. Barrow, Deterioration of Book Stock, Causes and Remedies, ed. Randolph W. Church (Richmond, Va.: The Virginia State Library, 1959), 15.

3. Ibid., 577 .

4. Ibid., 579 .

5. Brian J. Baird, et al., "Findings from the Condition Surveys Conducted by the University of Kansas Libraries," College E Research Libraries 58 (Mar. 1997): 115.

6. Task Force on Preservation, "University of Illinois at Urbana-Champaign, Collection Analysis Project: Report of the Task Force on Preservation" (Urbana: Univ. of Illinois at UrbanaChampaign, 1980, photocopy) and Illinois Library Materials Preservation Task Force, "The Preservation of Library and Archival Resources in Illinois: A Five-year Plan of Action" (Carbondale, Ill.: Illinois Library Materials Preservation Task Force, 1986).

7. First published in 1982, the Association of Research Libraries' (ARL) Preservation Planning Program: An Assisted Self-study Manual for Libraries encouraged libraries to systematically study their collections and facilities through a process of assessment. In 1987, Pamela W. Darling expanded the publication and Wesley Boomgaarden revised the resource notebook to include significant selections from the burgeoning preservation literature. In 1986-1987, Northwestern University, the Colorado State University Libraries, Ohio State University, and many other institutions published these reports. 
8. Bradley L. Schaffner and Brian J. Baird, "Into the Dustbin of History? The Evaluation and Preservation of Slavic Materials," College \& Research Libraries 60 (Mar. 1999): 144.

9. The 1989 UIUC assessment and the 1982 Stanford assessment upon which it was based both excluded unbound serials.

10. Buchanan and Coleman, "Deterioration Survey of the Stanford University Libraries

Green Library Stack Collection."

11. The UIUC 1989 assessment evaluated books according to these criteria: condition of the paper, condition of the binding, and condition of the external covers. The books received a score for each category, and the overall score for each book was determined based on the combination of the three scores. Before the final score was assessed, the surveyors were instructed to give paper condition twice the weight of binding or external covers. The rationale for this decision was that paper represents the intellectual content of the book and thus the authors surmised that the library would be most interested in its condition.

12. AZPlanSite is located at http://www.azplansite.com/samplesize.htm and was last updated on June 6, 2002. It includes the online sample calculator and a brief explanation of its equations. For further elaboration on determining sample size, please refer to Ronald Czaja and Johnny Blair, Designing Surveys: A Guide to Decisions and Procedures (Thousand Oaks, Calif.: Pine Forge Pr., 1996), 126-33.

13. John Dean, "Managing Collection Information for Preservation Planning," in Advances in Preservation and Access, vol. 2, ed. Barbara Higgenbotham (Medford, N.J.: Learned Information, Inc., 1995), 206. Although John Dean's article emphasized the significant issues with excluding such nonrespondents from surveys and assessments, our statistician's assessment was that an adequate sample size was generally based on respondents in relation to the population, not the population itself.

14. Chrzastowski, et al., "Library Collection Deterioration," 580.

15. Oversized pieces are between ten and a half and seventeen inches. Folios are pieces that are seventeen inches or higher. Undersized pieces measure less than six inches.

16. Chrzastowski, et al., "Library Collection Deterioration," 580.

17. The call numbers in the West stacks in 2002 are the same call numbers moved in 1984. Thus, books in the collection during the original move have benefited from the climate-controlled environment for eighteen years. The move is described in the library's newsletter: Ruth McBride, "Central Bookstacks: Progress Report," Library Office Notes, no. 30 (July 1984).

18. John DePew, A Library, Media, and Archival Preservation Handbook (Santa Barbara, Calif.: ABC-CLIO, 1991): 298-99. The figures provided by the library's consultant were taken from those cited in DePew's volume and adjusted for 2000 dollars.

19. Jeanne M. Drewes and Julie A. Page, Promoting Preservation Awareness in Libraries: A Sourcebook for Academic, Public, School, and Special Collections (Westport, Conn.: Greenwood Pr., 1997).

20. Chrzastowski, et al., "Library Collection Deterioration,” 580. 